\title{
Diagnostic set-up and modelling for investigation of synergy between 3D edge physics and plasma-wall interactions on Wendelstein 7-X
}

Y. Liang ${ }^{1, \mathrm{a}}$, O. Neubauer ${ }^{1}$, R. König ${ }^{2}$, M Krychowiak ${ }^{2}$, B. Schweer ${ }^{3}$, P. Denner ${ }^{1}$, M. Rack ${ }^{1}$, D. Reiter ${ }^{1}$, Y. Feng ${ }^{2}$, A. Krämer-Flecken ${ }^{1}$, P. Drews ${ }^{1}$, F. Hasenbeck ${ }^{1}$, S. Liu ${ }^{1,4}$, Y. Gao ${ }^{1,4}$, E. H. Wang ${ }^{1}$, Y. Wei ${ }^{1}$, M. Dostal ${ }^{1}$, L. Li ${ }^{1,5}$, N. Wang ${ }^{1,6}$, J. Geiger ${ }^{2}$, Y. Suzuki $^{7}$, S. Sereda ${ }^{1}$, P. Börner ${ }^{1}$, A. C. Weger ${ }^{1}$, W. Biel ${ }^{1}$, S. Brezinsek ${ }^{1}$, A. Charl ${ }^{1}$, G. Czymek $^{1}$, D. Höschen ${ }^{1}$, F. Effenberg ${ }^{8}$, O. Grulke ${ }^{1}$, D. Nicolai ${ }^{1}$, H. T. Lambertz $^{1}$, O. Marchuk ${ }^{1}$, O. Schmitz ${ }^{8}$, K. P. Hollfeld ${ }^{1}$, M. Knaup ${ }^{1}$, G. Offermanns ${ }^{1}$, G. Satheeswaran ${ }^{1}$, A. Terra ${ }^{1}$, J. Thomas ${ }^{1}$, T. S. Pederson ${ }^{2}$, U. Samm ${ }^{1}$, C. Linsmeier ${ }^{1}$ and the W7-X team

${ }^{1}$ Forschungszentrum Jülich GmbH, Institut für Energie - und Klimaforschung - Plasmaphysik, Partner of the Trilateral Euregio Cluster (TEC), 52425 Jülich, Germany

${ }^{2}$ Max Planck Institute for Plasma Physics, 17491 Greifswald, Germany

3 Laboratoire de Physique des Plasmas - Laboratorium voor Plasmafysica, ERM/KMS, 1000 Brussels, Belgium

${ }^{4}$ Institute of Plasma Physics, Chinese Academy of Sciences, Hefei 230031, China

${ }^{5}$ College of Science, Donghua University, Shanghai 201620, China

${ }^{6}$ State Key Laboratory of Advanced Electromagnetic Engineering and Technology, Huazhong

University of Science and Technology, Wuhan, Hubei 430074, China

${ }^{7}$ National Institute for Fusion Science, Japan

${ }^{8}$ University of Wisconsin - Madison, Madison, WI, USA

${ }^{a}$ Contact Email: Y. Liang@fz-juelich.de

Abstract: A group of edge diagnostics and modelling has been developed for investigation of synergy between $3 \mathrm{D}$ edge physics and plasma-wall interactions on Wendelstein 7-X (W7-X). Two endoscopes have been designed for visible and ultraviolet spectroscopy and tomography of the plasma edge, along with infrared thermography of the divertor tiles. Two-dimensional profiles of impurities (e.g. helium, carbon) will be measured by two endoscopes viewing the island divertor region in the plasma edge with a spatial resolution of $<2 \mathrm{~mm}$. A multipurpose manipulator, which is used as the carrier either of the probe head for measuring the plasma edge profiles or of samples for plasma exposure studies, was installed at the outside midplane on $\mathrm{W} 7-\mathrm{X}$ in 2015. A poloidal correlation reflectometer has also been installed at W7-X. The system consists of an antenna array observing the propagation of turbulent phenomena in the mid-plane. The EMC3-EIRENE code package has been adapted for plasma edge transport 
in helium plasma at W7-X using a hybrid fluid-kinetic approach by enabling EMC3 to treat non-hydrogen isotopes and extending the usage of EIRENE features within EMC3-EIRENE.

\section{Introduction}

Steady-state operation of future fusion power plants requires a solution for a tolerable plasma exhaust, including steady-state and transient heat and particle fluxes on plasma-facing components. Recently, applications of the three-dimensional (3D) magnetic topology for controlling the edge plasma transport, stability, and plasma-wall interactions (PWIs) have attracted much attention in fusion research, especially the use of resonant magnetic perturbation coils in tokamaks [1]. To investigate this physics issue, however, it is very likely that a superconducting stellarator device, which allows a long-pulse stable operation with an intrinsic 3D magnetic topology concept, will be of great benefit.

The superconducting stellarator Wendelstein 7-X (W7-X) [2], a drift-optimized stellarator with improved neoclassical confinement, is designed for an steady-state plasma operation with a maximum magnetic field strength on axis of $3 \mathrm{~T}$, a major radius of $\mathrm{R}_{0}=5.5 \mathrm{~m}$ and an effective minor radius of $<\mathrm{a}>=0.55 \mathrm{~m}$. One major objective of the $\mathrm{W} 7-\mathrm{X}$ experiment is to demonstrate steady-state divertor operation at high densities and high central temperatures. Therefore, investigation of the role of 3D divertor concepts in the physics and control of edge transport and stabilities, heat and particle exhausts is essential.

In fact, plasma operation of W7-X follows a staged approach following the successive completion of the in-vessel components. Starting with a limiter configuration, the first W7-X experimental campaign OP1.1 has been successfully carried out. The experiments in OP1.1 were operated in helium $(\mathrm{He})$ in the beginning of the campaign and in hydrogen $(\mathrm{H})$ in the late phase. The second campaign OP1.2 has been scheduled for 2017. In this phase, plasma operation with a divertor configuration without water cooling will be implemented, and the total heat power will be upgraded from 5MW (electron cyclotron resonance heating (ECRH) only) to about $20 \mathrm{MW}$ (ECRH: 8.8MW, neutral beam injection (NBI): $10 \mathrm{MW}$ (deuterium (D))/ $7 \mathrm{MW}(\mathrm{H})$, ion cyclotron resonance heating (ICRH): 1.6MW). The material to be used for W7-X plasma facing components (PFC) is carbon (C). The maximum pulse length is expected to be $\sim 10$ s with a total heating power of $\sim 10 \mathrm{MW}$, and up to $\sim 60$ s with reduced power in this OP1.2 phase. The main goal of OP1.2 is the preparation of the steady-state phase, which has been planned in OP2 [3].

\section{Synergy between $3 D$ edge physics and plasma-wall interactions on W7-X}


The concept of W7-X for plasma exhaust uses the formation of an inherent separatrix at the boundary for creating a so-called island divertor [4]. The confinement region is either limited by the separatrix of the boundary island chain or by an ergodized region formed by the remnants of overlapping high-order rational islands around the major resonance. Three major island divertor configurations with rotational transform values of $\imath=5 / 6,5 / 5$ and $5 / 4$ are available for use in the coming W7-X experimental campaign OP 1.2.

The PWI in the divertor region of W7-X will be of great importance for the operational phase OP1.2. While the erosion of the divertor will have an impact on its lifetime and is therefore a critical subject of investigation, fundamental PWI studies in the divertor region are in many ways equally significant. These PWIs will be influenced by impurity transport,

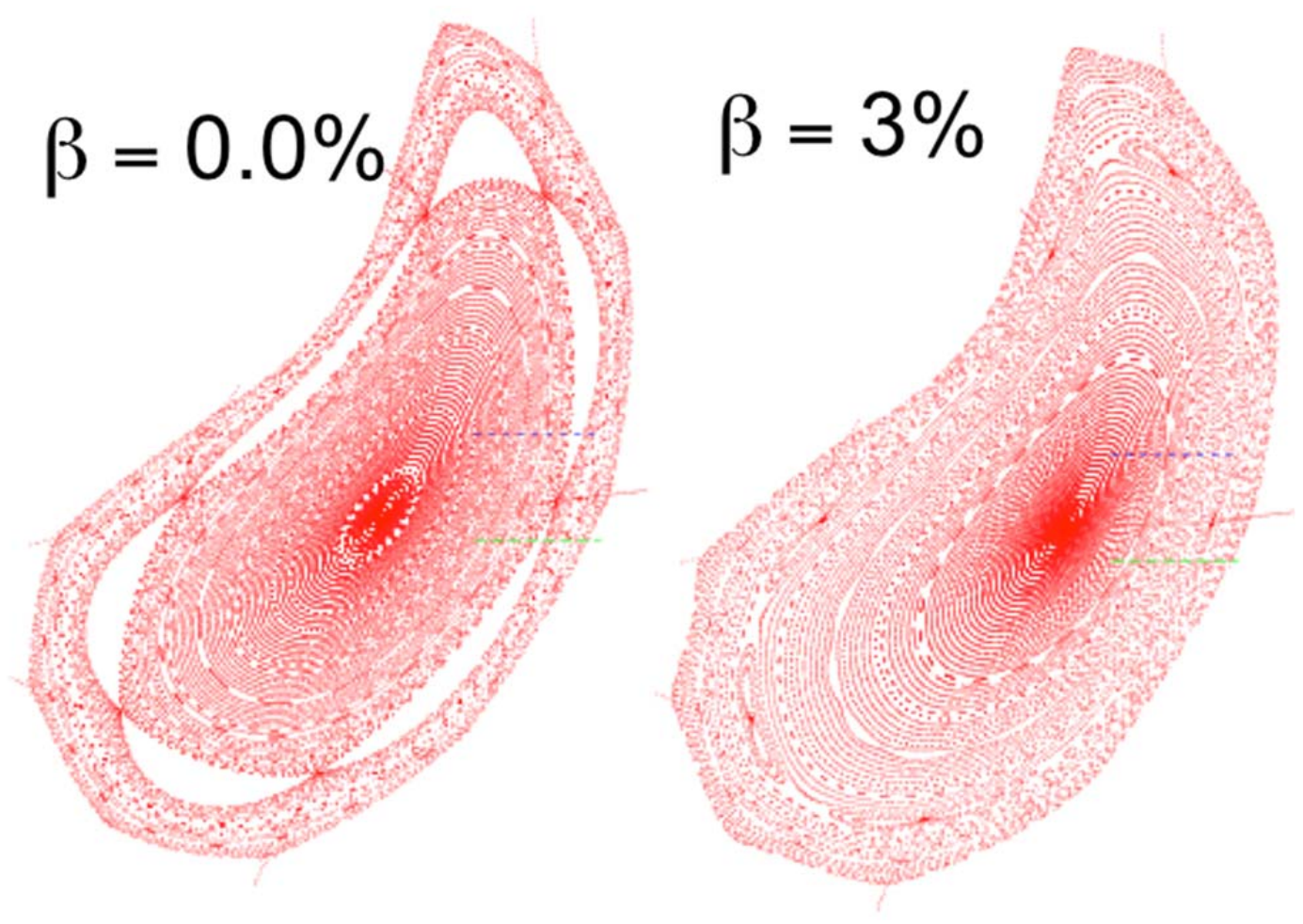

Figure 1 Beta dependence of the magnetic topology on W7-X. Here, the 3D equilibrium is calculated by the HINT2 code with a low-iota configuration. The magnetic islands are $\mathrm{n} / \mathrm{m}=5 / 6$.

where the complex 3D magnetic geometry will play a crucial role, but also the magnetic geometry itself will be influenced by plasma effects such as Pfirsch-Schlüter and bootstrap currents. In Fig. 1, the results calculated by the 3D equilibrium code HINT2 indicate that the width of the edge islands reduces gradually with an increase in plasma beta from $0 \%$ (vacuum assumption) to $3 \%$. Here, $3 \%$ is the maximum value of plasma beta contemplated in OP1.2. This will directly influence the distribution of the field line connection length and the 
performance of the island divertor. Furthermore, the edge transport and stability, impurity screening effect, and plasma-wall interactions will be affected self-consistently. Therefore, along with measurements of obvious quantities such as heat flux, PWI research in the divertor region will also require measurements of the temperature in the plasma edge and of the concentration and distribution of different impurities, in combination with modelling of impurity transport.

\section{Diagnostic set-up and modelling for $3 \mathrm{D}$ edge physics and plasma-wall interactions}

To investigate systematically the synergy between 3D edge physics and PWI, a set of edge diagnostics (see Fig. 2) has been developed and the EMC3-EIRENE code is being extended to He plasmas. In particular, both, poloidal correlation reflectometer and the mid-plane manipulator equipped with a combined probe head have been successfully applied for providing characteristics of the $3 \mathrm{D}$ edge layer with plasma density, temperature and rotation profiles during the OP1.1 phase. The divertor gas inlet system has been used for the helium

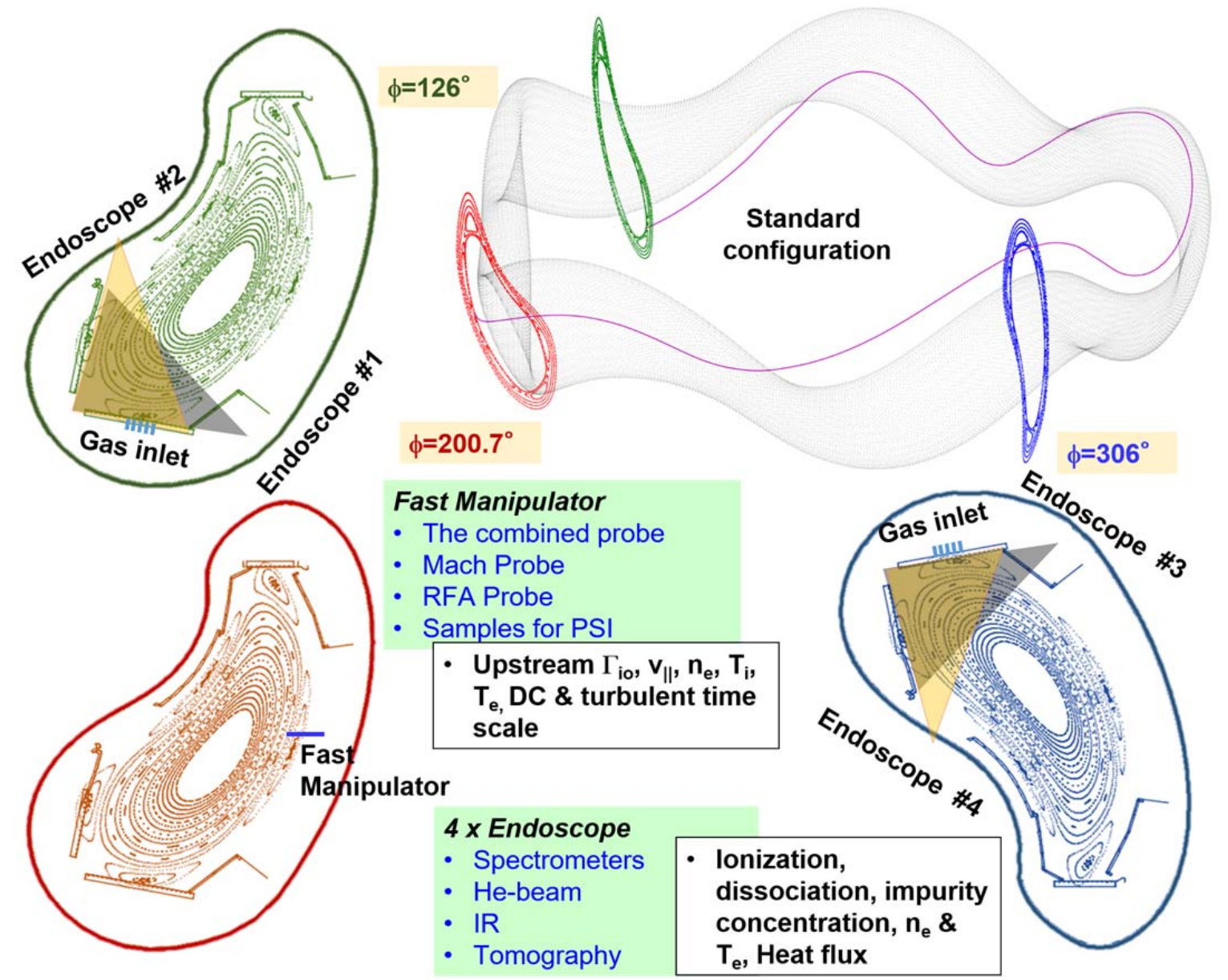

Figure 2 Toroidal distribution of the edge diagnostics for investigation of synergy between 3D edge physics and plasma-wall interactions on $\mathrm{W} 7-\mathrm{X}$. The upper right plot shows a magnetic field line starting from the frontend of the fast manipulator at $\phi=200.7^{\circ}$ to the toroidal sections at $\phi=306^{\circ}$ and $\phi=126^{\circ}$, where two identical sets of divertor endoscopes and gas inlets to be located at. Here, the magnetic topology plotted are corresponding to the standard $\mathrm{l}=5 / 5$ island divertor configuration. 
beam diagnostics and the impurity seeding experiments on W7-X in the beginning of 2016. Furthermore, the divertor endoscope system has been optimized to get rid of the ghost image with a wedge-shaped filter. The first two divertor endoscopes viewing the lower divertor unit will be installed at the toroidal section $\left(\phi=126^{\circ}\right)$ on $\mathrm{W} 7-\mathrm{X}$ for the experimental campaign OP1.2, while the last two viewing the upper divertor unit will be installed at the opposite toroidal section $\left(\phi=306^{\circ}\right)$ before the OP2 starts. In addition to the diagnostic setup presented in ref. [5], several new probe heads, including an improved combined probe, a dedicated Mach probe array, and a multiple channel retarding field analyser (RFA) probe, have been developed for W7-X, and they will be operational for the OP1.2 phase. Several spectrometers, filter cameras and infrared cameras have been established and calibrated, and they will be applied for the upcoming OP1.2 experiments. In this section, an overview of those advanced progresses on the development of diagnostic set-up and modelling for 3D edge physics and plasma-wall interactions will be presented.

3.1 Measurements of electron temperature, density, and impurity distributions in the divertor region, and heat flux on the local divertor target plate

In order to study the origin of impurities ( $\mathrm{C}$ is expected to dominate in OP1.2) and edge impurity transport with different magnetic configurations of island divertor on W7-X, a set of endoscopes has been designed for visible and ultraviolet spectroscopy and for tomography of the plasma edge, along with infrared thermography of the divertor tiles [6]. Two of them viewing a lower divertor unit will be available during the OP1.2 phase. The optic focus is chosen at the location of the gas nozzles while an entrance aperture of approximately $6 \mathrm{~mm}$ in diameter ensures an appropriate depth of field. The small entrance aperture in the actively cooled front end of the endoscope tube limits the maximum temperature of the first mirror assembly during steady state operation. Two-dimensional profiles of impurities (e.g. He, C) will be measured by two endoscopes viewing the island divertor region in the plasma edge with a spatial resolution of $<2 \mathrm{~mm}$. The working spectral range of each endoscope is from $350 \mathrm{~nm}$ to $7000 \mathrm{~nm}$. The light from the divertor plasma is divided equally into two branches by a prism in the detector box. Each branch is divided into ultraviolet (UV: $350 \mathrm{~nm}-550$ $\mathrm{nm}$ ), visible (VIS: $550 \mathrm{~nm}-950 \mathrm{~nm}$ ) and infrared (IR: $950 \mathrm{~nm}-7000 \mathrm{~nm}$ ) sub-branches by dichroic filters. The ultraviolet and visible sub-branches are divided again for the filter cameras and spectrometers.

Each endoscope includes 5 filter cameras (2 UV and 3 VIS filter cameras), 1 infrared camera and 5 spectrometers ( 2 UV, 1 VIS, 1 IR and 1 overview spectrometer). The image 
size in the focal plane is $14 \mathrm{~mm} \times 14 \mathrm{~mm}$, which is focused onto 14 fibers (diameter $1 \mathrm{~mm}$ ) in an array by a cylindrical lens. The 14 fibers are connected to the spectrometer located in a laboratory. The spatial resolution on the divertor plates is $2 \mathrm{~cm}-3 \mathrm{~cm}$. Two UV spectrometers use high- $n$ Balmer spectroscopy to deduce electron density from Stark broadening, and electron temperature from line ratio or discrete-to-continuum transition. These two UV spectrometers could also be used to measure the CD Gerö and C2 Swan bands in order to study the chemical sputtering on the carbon divertor plates. The VIS spectrometer is used to measure the Fulcher band in order to study the hydrogen recycling on the divertor plates. The VIS spectrometer could also be used to measure helium lines in order to deduce electron density and temperature. The IR spectrometer is used to measure the Paschen series in order to deduce electron temperature and density.

An overview spectrometer, which can cover a wide wavelength range to monitor impurity contents in the divertor region, is linked to full-spectrum fibers located at the leading vertex of the pyramidal mirror in the endoscope system. The light from the plasma will be carried by several $1 \mathrm{~mm}$ diameter fibers to the diagnostics room, where it will be coupled to the fivechannel overview spectrometer (Avantes model: AVASPEC-ULS2048L-USB2-RM). Each channel is a miniature Czerny-Turner spectrometer with a fixed grating to cover a certain wavelength range. Together, these channels can cover the wavelength range from 300 to $1100 \mathrm{~nm}$. This overview spectrometer will be used for routine investigation of the impurity contents under different operational scenarios. It will also provide reference data for other diagnostic equipment on the endoscope system, with which it shares the same field of view.

Through a pair of endoscopes, a high-resolution divertor infrared (D-IR) thermography system, which includes two medium-wavelength range $(3-5 \mu \mathrm{m})$ infrared cameras, will be implemented on $\mathrm{W} 7-\mathrm{X}$ to monitor the temperature and to measure the heat flux on both the vertical and horizontal divertor targets in one toroidal cross section during the campaign OP 1.2. This D-IR system has a high spatial resolution of less than $2 \mathrm{~mm}$ on the divertor target plate, and it will allow for a detailed study of the footprint patterns. With an optimized smaller field of view, measurements with high temporal resolution could be achieved to investigate transient events (i.e. Edge localized Modes) releasing large amounts of energy. The designed rotating mirror at the front-end of the endoscope enables poloidal scanning of the region of interest, thus the viewing area of the camera has the potential to be actively shifted during the operation for the strike line characterization under different configurations.

The whole endoscope system is currently under construction and will be installed on W7-X. A similar system was successfully installed on JET [7] (the world's largest tokamak) in 2012. 
One $0.75 \mathrm{~m}$ imaging Czerney-Turner spectrometer and two $0.75 \mathrm{~m}$ Littrow spectrometers will be available at the start of OP1.2. In addition, a high-efficiency XUV overview spectrometer (HEXOS) has been used for monitoring impurity concentration in the plasma core.

\subsection{Measurement of edge upstream plasma profiles}

A multi-purpose manipulator, which is used as the carrier either for measurements of the plasma edge profiles or for plasma exposure studies, was installed at the outside mid-plane on W7-X in $2015[8,9]$. One of the most important features of the manipulator is the fast and stable movement of the probe. The maximum acceleration and velocity are $3 \mathrm{~g}$ and $3.5 \mathrm{~m} / \mathrm{s}$, respectively. A combined probe head, which measures the radial distribution of the magnetic field with magnetic pick-up coils, the plasma temperature and density profiles with Langmuir probes and the plasma flows with a Mach probe, was mounted on the manipulator and commissioned during the campaign OP 1.1 [10]. For the next operational campaign, an
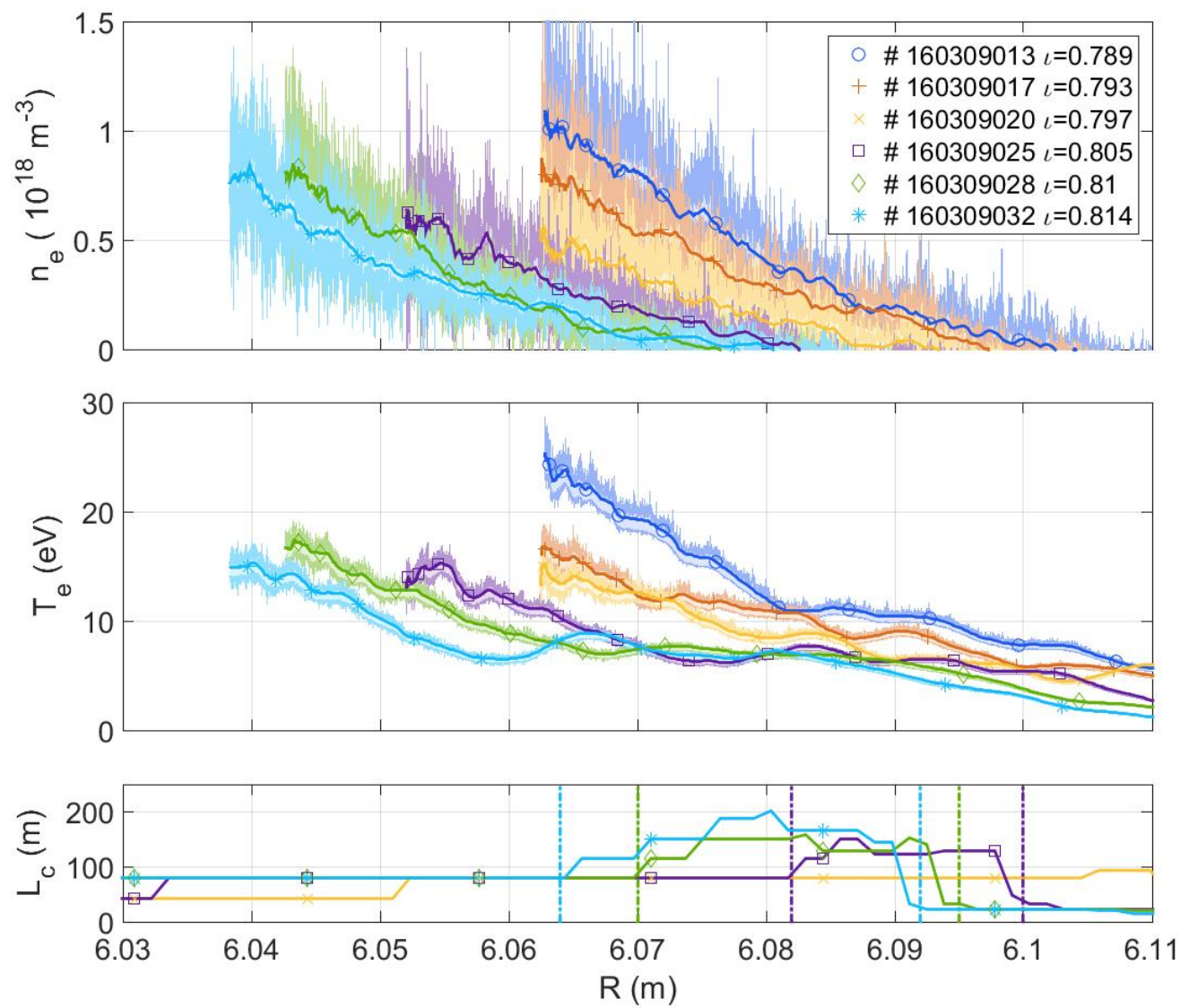

Figure 3 Edge profiles of electron density $\left(n_{e}\right)$ and temperature $\left(T_{\mathrm{e}}\right)$ measured by the combined probe, and the field line connection length on $\mathrm{W} 7-\mathrm{X}$ with various inwardly shifted high-iota limiter configurations. Here, the measurements are performed in OP1.1, and the error bars defined by the standard deviation of the measured signals are also shown. The flat area appearing in the temperature profile might be due to the edge islands indicated by the change of field connection length. 
improved design of the combined probe, a retarding field analyser (RFA) and a dedicated Mach probe array will be employed. For plasma wall interaction studies, the ability to expose samples on the combined probe and on a dedicated heatable probe holder is planned.

The combined probe yielded good results for the edge profiles of the radial electric field, electron temperature and density and the magnetic field in the first campaign. The probe was able to measure both during a plunge and in a stationary position at the plasma edge. A series of scenarios with a tuning of the planar coils and a ramp of the iota was carried out. In this experiment, the measurements were undertaken under the same ECRH heating power of 2.7 MW. The combined probe is measuring upstream values of up to a maximum of $25 \mathrm{eV}$ (see Fig. 3). The deepest point of measurement was about $2 \mathrm{~cm}$ away from the last closed flux surface. While the limiter Langmuir probes yield downstream values of between $25 \mathrm{eV}$ and $40 \mathrm{eV}$. These measurements showed that the combined probe is able to reliably measure the edge parameters.

For plasma edge characterization and measurements of plasma parameters, particularly ion temperature, using an RFA [11] is a well-known technique which has shown good results on various fusion devices. For W7-X, the combined RFA probe head contains two Langmuir pins to measure electron parameters and 2 back-to-back oriented RFA modules to measure the ion parameters simultaneously, each with 3 independent, radially shifted channels (see Fig. 4). This combined RFA probe will be mounted on the fast multi-purpose manipulator on W7- X, so that it is possible to determine ion parameter profiles through the SOL up to several $\mathrm{cm}$ inside the separatrix. By adjusting the applied grid potentials, it is not only possible to determine ion parameters but also to use the probe head for multi-channel electron parameter measurements or high-energy particle loss measurements. Here, the physics design of two back to back oriented RFA modules is aiming for the measurement of fast particle loss which comes from one direction to the probe [12]. 
This probe head has a spatial resolution of $100 \mu \mathrm{m}$ and a radial distance between the channels of $3 \mathrm{~mm}$. To ensure that the probe head is always oriented along the magnetic field lines, independent of the chosen magnetic field configuration, it is possible to rotate the probe head manually adjusting the alignment within a range of 10 degree and with an accuracy of

0.5
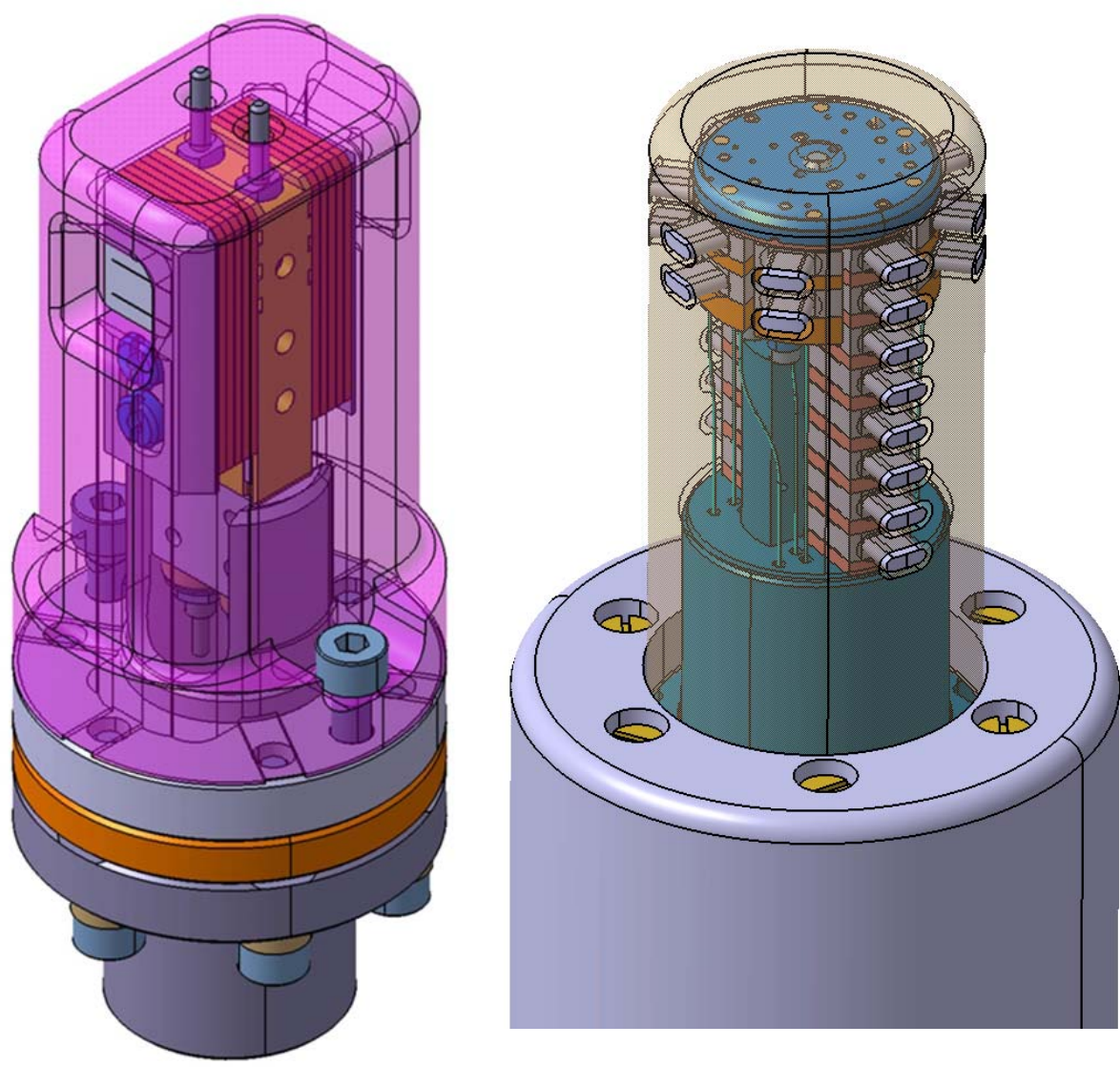

Figure 4 Sketches of the combined RFA probe (left) and the Mach probe (right) for W7-X

thermal loads, the slit plate for particle flux reduction inside the probe head and for dividing the particle flux into the various channels and also the biased grids are made of tungsten. However, the cover is made of graphite to reduce overall the probe weight. The design of the combined RFA probe has been finalized. The first experiments are scheduled for the OP1.2 at W7-X.

To study the dynamics of edge flow profiles with different heating, gas fuelling or impurity seeding in plasmas with different magnetic topology, a multi-array Mach probe, which includes 8 rows in the radial direction and 28 pins in total, is under construction for W7-X. Each of the top two rows includes 8 pins evenly distributed around the cylindrical probe, while other six rows include 2 back-to-back pins each, and they are oriented along the magnetic field lines. Each probe pin is made of tungsten and has a collecting area of $12 \mathrm{~mm}^{2}$. Moreover the multi-array Mach probe measures edge profiles of particle fluxes with a high 
temporal resolution of few hundred $\mathrm{kHz}$. Therefore, the characterization of edge transport for transient events is possible.

It should be noted that both, the combined RFA probe and the multi-array Mach probe, are featured with independent, radially shifted channels along the probe head. They are capable of investigating the radial heat and particle propagations as well as dynamic of the edge profiles during a transit event, such as the edge localised mode.

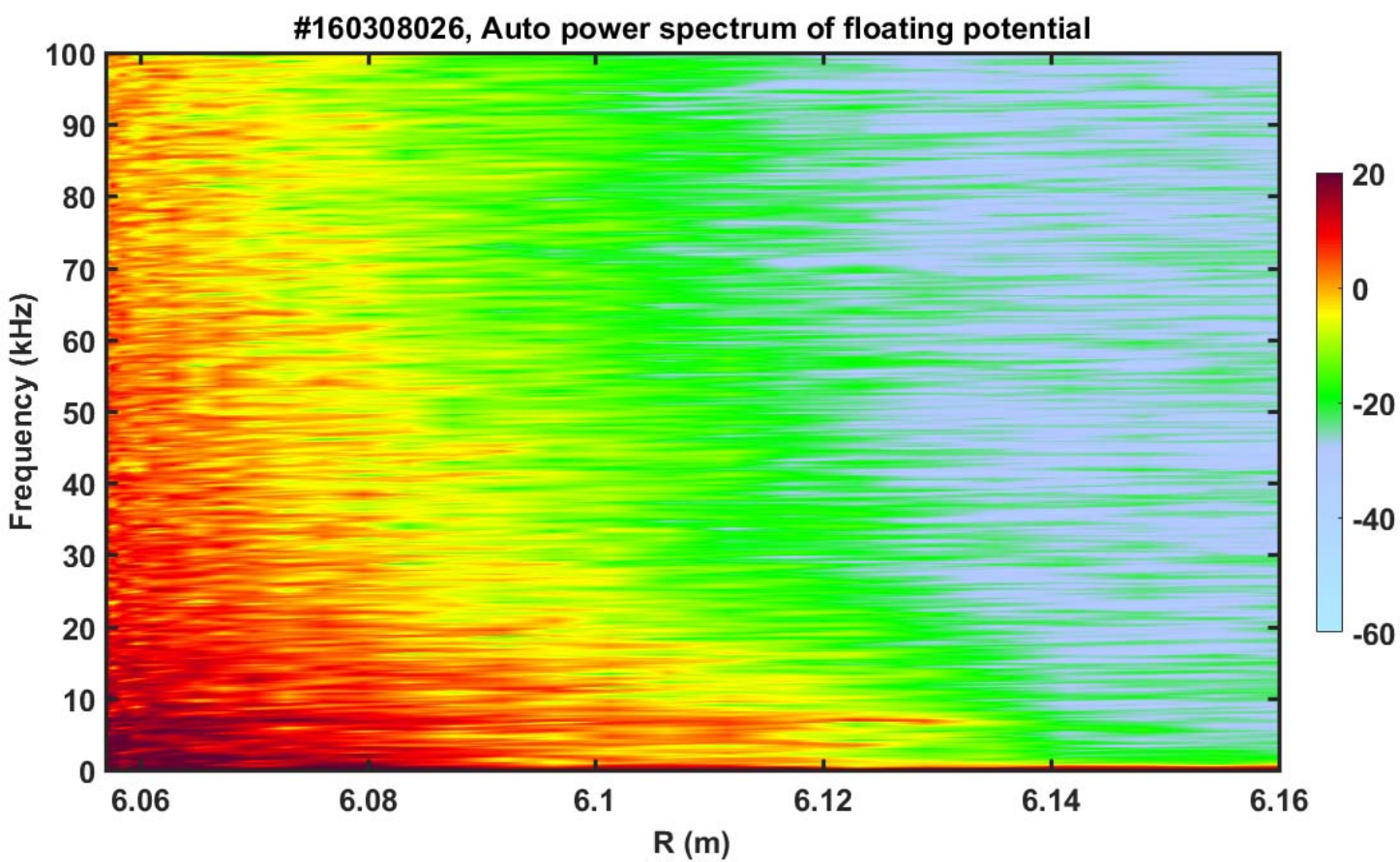

Figure 5 Auto power spectrum of the edge floating potential measured by Langmuir probes on $\mathrm{W} 7-\mathrm{X}$ in the limiter configuration.

\subsection{Measurements of edge turbulence and the pitch angle of field lines}

Edge turbulence characteristics can be obtained by the Langmuir probes on the combined probe head, such as turbulence decorrelation time, cross correlation relationship and turbulence propagation along both the poloidal and radial directions, along with phase velocity, particle flux driven by turbulence and Reynolds stress. During the campaign OP1.1, the typical poloidal correlation length of turbulence was about $0.5-1 \mathrm{~cm}$ in the SOL region. Figure 5 shows a typical auto power spectrum from the floating potential measured by Langmuir probes, with line averaged density $<\mathrm{n}_{\mathrm{e}}>=2.4 \times 10^{19} \mathrm{~m}^{-3}$ and ECRH heating power $\mathrm{P}=4 \mathrm{MW}$ in the standard limiter configuration. A mode at frequency of $\sim 7 \mathrm{kHz}$ is observed as shown in Fig. 5. This mode can be detected at $\mathrm{R}=6.15 \mathrm{~m}$, about $13 \mathrm{~cm}$ outside of the last 
closed flux surface (LCFS), and its amplitude increases significantly when close to the separatrix. It should be noted that the magnetic coils embedded in the combined probe head also measured this $7 \mathrm{kHz}$ mode.

A poloidal correlation reflectometer has been installed at W7-X [13]. The system consists of an antenna array observing the propagation of turbulent phenomena in the mid-plane. The diagnostic operates in a bean shaped poloidal plasma cross section. The five antennae aim at the plasma edge $\left(\mathrm{R}=6 \mathrm{~m}, \mathrm{Z}=-0.104 \mathrm{~m}\right.$ and $\left.\Phi=71.05^{\circ}\right)$. The system is operated in O-mode polarization, which limits the observation range to those reflection layers $\left(r_{c}\right)$ which fulfil the condition $0.6 \times 10^{19} \mathrm{~m}^{-3}<\mathrm{n}_{\mathrm{e}}\left(\mathrm{r}_{\mathrm{c}}\right)<2 \times 10^{19} \mathrm{~m}^{-3}$. Beside the spectral characterization of the turbulence, the system is capable of measuring the poloidal propagation of the turbulence from 6 different antenna combinations.

Moreover, the poloidal and toroidal separation of the system allows the inclination angle of the turbulence to be measured. Assuming that the turbulence is aligned to the magnetic field line, the pitch of the magnetic field line can be calculated. Deviation from the magnetic field line pitch can be considered to be caused by the limited toroidal correlation length of the investigated turbulence. Furthermore, measurements of the pitch will be attempted in the vicinity of the island divertor. The pitch is related to the iota in the island. Accessing this quantity enables the measurement of the topological parameter relevant for the flux diversion: the field line connection length. In the island divertor of W7-X, this length is calculated from the internal rotational transform inside the island.

Another relevant topic is the existence of zonal flows. These flows are characterized in that a temporal increase in the flow velocity can be determined by the instrument. To be able to measure transient events with sufficient frequency for averaging, the temporal resolution of the diagnostic is $4 \mathrm{MHz}$. This should allow the measurement of velocities in less than $1 \mathrm{~ms}$. The system has been in operation since December 2015, and data are taken on a daily basis. In addition, the reflected signal is used as indication of the achieved plasma density, because the reflection condition depends on the local plasma density.

\subsection{Modelling for 3D Edge transport and heat load distributions}

Understanding the plasma exhaust processes in stellarators requires 3D transport models that are capable of dealing with transport processes in a stochastic field where closed flux surfaces do not exist. For this, the 3D plasma edge Monte Carlo code EMC3 will be used in connection with the W7-X island divertor experiments. 
The fluid edge plasma MonteCarlo code in three dimensions (EMC3) [14] coupled to the kinetic (neutral) transport code EIRENE $[15,16]$ is a commonly used plasma edge simulation code for treating complex magnetic configurations. EMC3EIRENE has been continuously improved and verified, but one remaining restriction up to now had been that the bulk ion species was limited to hydrogen isotopes, with higher- $Z$ ions being treated as trace-impurities.

However, in initial operational phases, W7-X was operated with helium plasma. Computational quantification of helium plasma flows in the edge is required to extend the understanding of the involved physical processes. Therefore, an approach was studied to simulate helium plasma by slightly extending EMC3 to facilitate a treatment of the main plasma species with $\mathrm{Z}$ $\neq 1$ and expanding the use of EIRENE features within EMC3.

A zero dimensional estimate of the ionization and population

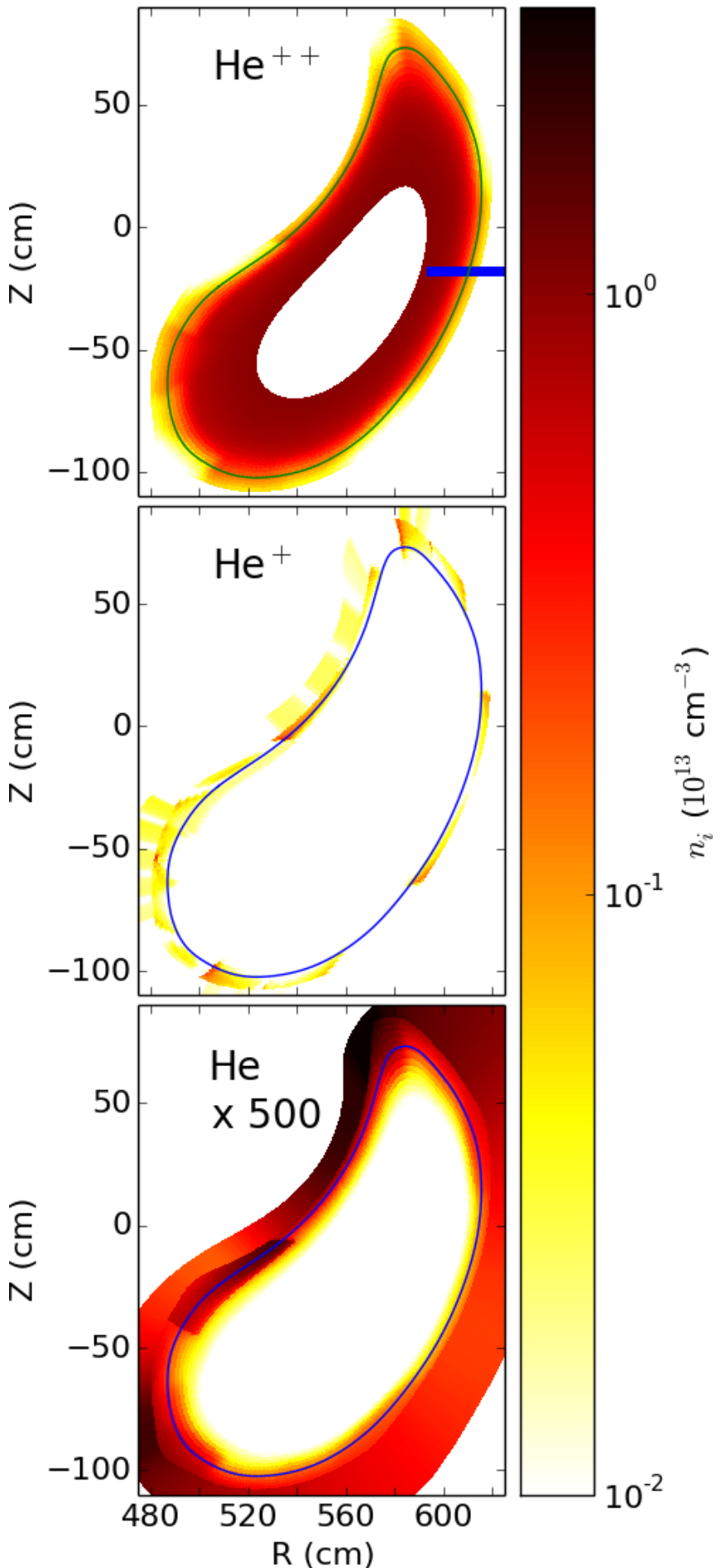

Figure 6 Comparison of neutral helium, $\mathrm{He}+$, and $\mathrm{He}++$ density distributions calculated for a typical W7-X limiter plasma with an averaged upstream ion density of $\left\langle\mathrm{n}_{\mathrm{i}}\right\rangle_{\mathrm{LCFS}}=0.5 \times 10^{13} \mathrm{~cm}^{-3}$ and a heating power of $\mathrm{P}=$ $2 \mathrm{MW}$. The blue line indicates the location of the multipurpose manipulator and the solid line represents the LCFS. distributions using collisional-radiative rate coefficients by McWhirter and Hearn [17] and Fujimoto [18] as well as estimated transport losses (via HYDKIN [19]) indicates that a 
reasonable treatment of helium plasma as a single-fluid system with helium in the second ionisation state is justified for limiter plasmas at W7-X $\left(\mathrm{n}_{\mathrm{e}}>1 \times 10^{13} \mathrm{~cm}^{-3}\right.$ and $\left.\mathrm{T}_{\mathrm{e}}>20 \mathrm{eV}\right)$.

A second estimate for the correct treatment of helium plasma can be made based on EIRENE test simulations by comparing the recycling flux from the targets with fluxes from volume-recombination. The simulations show that the dominant source for $\mathrm{He}^{+}$ions under the given conditions is ionization from helium atoms rather than volume-recombination from $\mathrm{He}^{++}$ions. Thus, it leads to the same conclusion as the zero-dimensional estimate: helium in the second ionization state is dominant in the plasma edge. Furthermore, this can be strengthened by comparing the simulated spatial distribution of neutral helium, $\mathrm{He}^{+}$, and $\mathrm{He}^{++}$ in a typical limiter discharge at W7-X (see Fig. 6). Here, these simulation results were shown for a poloidal cross-section similar to that seen by the multi-purpose manipulator. The edge profiles measured by the probes will be used to constrain the EMC3-Eirene simulations.

A treatment of neutral helium and $\mathrm{He}+$ ions is also required for a sufficient consideration of plasma wall interactions, which can be performed using the kinetic transport code EIRENE. In the simplest approach, the $\mathrm{He}^{+}$ions can be considered to have a distribution function that is a general solution of the Boltzmann equation. Therefore, an adequate treatment of $\mathrm{He}^{+}$ions is drift kinetic, incorporating transport effects at least at a certain level. The simplest transport model employed consists of following the $\mathrm{He}^{+}$ions in the test particle picture. Trajectory integration is carried out in the guiding centre approximation, ignoring drift motion or anomalous transport. Processes like $\mathrm{He}-\mathrm{He}^{+}$charge-exchange are considered via the nonlinear collision model of EIRENE. The dominant ion species $\mathrm{He}^{++}$is treated with the fluid approach using a generalized model for EMC3 allowing for non-hydrogen isotopes.

Experiment-simulation comparisons of W7-X's first operation phase are currently ongoing and will be continued for the second operation phase of W7-X if helium operation will be performed (currently under discussion). Details on the model as well as sensitivity studies can be found in ref. [20]

\section{Summary and future plan}

Synergy between edge and divertor physics and plasma-wall interaction (PWI) will be of great importance for the operational phase OP1.2, in which various island divertor configurations will be applied for the first time on W7-X. These plasma-wall interactions will be influenced by impurity transport, where the complex 3D magnetic geometry will play a crucial role, but this magnetic geometry could itself be influenced by plasma effects. 
Therefore, in order to investigate systematically the synergy between 3D edge physics and the PWI, a set of edge diagnostics and modelling has been developed in combination with modelling of impurity transport on W7-X. The developed fluid-kinetic helium model enabled for a detailed comparison of various diagnostics in the first helium operation phase of W7-X and opens a new dimension for modelling of the non-active operation phase of ITER.

For future steady-state high performance experiments on W7-X in OP2 or beyond, several key edge physics and PWI issues, including divertor heat and particle exhausts and fuel retention, become more and more important. Various new edge diagnostics and modelling are under consideration in support of study of synergy between 3D edge physics and the PWI. A combined laser-induced breakdown / ablation spectroscopy (LIBS/LIAS) is currently under feasibility design for in-situ and real-time diagnostic of the co-deposition and fuel retention during and in between plasma discharges on W7-X. Based on the similar system developed on the TEXTOR tokamak [21] and the PSI-2 linear device [22], this system will be further optimised and to be available for OP2 on W7-X. A new fast divertor manipulator, using the similar technology of the mid-plane multi-purpose manipulator, is currently under consideration for both, measurement of divertor downstream profiles and exposure of material samples at the position few $\mathrm{mm}$ above the divertor plate.

Another important aspect of a long term experimental phases of Wendelstein $7-\mathrm{X}$ is to investigate the high power steady-state operation with alternative wall materials like tungsten. Apart from replacement divertor components the new fast divertor manipulator is available for exposure of material samples followed by in situ analysis in the target chamber and further analysis in material labs. The 3D Monte Carlo impurity transport code ERO [23], solving the kinetic equations of impurities in plasma in the vicinity of material surfaces, will be used for systematically investigating migration of eroded sample material to remote areas on W7-X.

\section{Acknowledgments}

This work has been carried out within the framework of the EUROfusion Consortium and has received funding from the Euratom research and training programme 2014-2018 under grant agreement No 633053. The views and opinions expressed herein do not necessarily reflect those of the European Commission. 
The authors gratefully acknowledge the computing time granted on the supercomputer JURECA [24] at Jülich Supercomputing Centre (JSC).

\section{References:}

[1] Liang, Y., et al., "Mitigation of type-I ELMs with n=1, 2 fields on JET with ITER-like wall", Nucl. Fusion 53 (2013) 073036;

[2] Wolf, R. C. et al., "Wendelstein 7-X Program-Demonstration of a Stellarator Option for Fusion Energy", IEEE TRANSACTIONS ON PLASMA SCIENCE, (2016)

[3] Bosch H.-S. et al., "Transition from construction to operation phase of the Wendelstein 7-X stellarator”, IEEE Trans. Plasma Sci. 42, 432, (2014)

[4] P Grigull, et al., "First island divertor experiments on the W7-AS stellarator", Plasma Phys. Control. Fusion, 43, A175-A193 (2001)

[5] Neubauer, O., et al., "Diagnostic setup for investigation of plasma wall interactions at Wendelstein 7-X”, Fusion Engineering and Design, 96-97 (2015) 891-894

[6] Neubauer, O., et al., "Optical Instruments for Local Divertor Observation at Wendelstein 7-X." 1st EPS Conference on Plasma Diagnostics (ECPD). 2015.

[7] A. Huber et al., "A new radiation-hard endoscope for divertor spectroscopy on JET”, Fusion engineering and design 88, 1361-1365 (2013)

[8] Nicolai, D., et al., "A Multi-Purpose Manipulator system for W7-X as user facility for plasma edge investigation",submitted to $29^{\text {th }}$ SOFT (2016)

[9] Satheeswaram, G., et al.,"A PCS7-based control and safety system for operation of the W7-X multi-purpose manipulator facility", submitted SOFT (2016)

[10] Drews, P., et al., "Measurement of the plasma edge profiles using the combined probe on W7-X". Preprint: 2016 IAEA Fusion Energy Conference, Kyoto [EX/P5-9] submitted to Nucl. Fusion.

[11] M. Kocan et al., "Measurements of ion energies in the tokamak plasma boundary", Journal of Nuclear Materials 415 (2011) S1133-S1138

[12] M. Rack, et al., "A rotating directional probe for the measurements of fast ion losses and plasma rotation at Tokamak Experiment for Technology Oriented Research", Review of scientific instruments, 84 (8), 083501 (2013)

[13] Krämer-Flecken, A., et al., "Investigation of turbulence rotation in limiter plasmas at W7-X with a new installed Poloidal Correlation Reflectometry”. Preprint: 2016 IAEA Fusion Energy Conference, Kyoto [EX/P5-4] submitted to Nucl. Fusion.

[14] Feng, Y., et al., "3D fluid modelling of the edge plasma by means of a Monte Carlo technique", Journal of Nuclear Materials, 266-269, 812 (1999).

[15] EIRENE. http://www.eirene.de. 
[16] Reiter, D., et al., “The EIRENE and B2-EIRENE Codes”, Fusion Science and Technology, 47

(2), $172(2005)$.

[17] McWhirter, R.W.P., et al., "Calculation of the Instantaneous Population Densities of the Excited Levels of Hydrogen-like Ions in a Plasma”, Proceedings of the Physical Society, 82 (5), 641 (1963).

[18] Fujimoto. T., "A collisional-radiative model for helium and its application to a discharge plasma", Journal of Quantitative Spectroscopy and Radiative Transfer, 21 (5), 439 (1979).

[19] HYDKIN. http://www.hydkin.de/.

[20] M. Rack, et al., "A fluid-kinetic approach for 3D plasma edge transport in He-plasma”, Nucl. Fusion, Accepted: 15 February 2017.

[21] N. Gierse, et al., "Time resolved imaging of laser induced ablation spectroscopy (LIAS) in TEXTOR and comparison with modeling”, Physica scripta, T167, 014034 (2016)

[22] X. Jiang, et al., "Design and development of a LIBS system on linear plasma device PSI-2 for in situ real-time diagnostics of plasma-facing materials", Nuclear Materials and Energy (2016), http://dx.doi.org/10.1016/j.nme.2016.11.021

[23] A. Kirschner, V. Philipps, J. Winter, U. Kögler, "Simulation of the plasma-wall interaction in a tokamak with the Monte Carlo code ERO-TEXTOR”, Nucl. Fusion 40 (2000) 989.

[24] Jülich Supercomputing Centre. (2016). JURECA: General-purpose supercomputer at Jülich Supercomputing Centre. Journal of large-scale research facilities, 2, A62.

http://dx.doi.org/10.17815/jlsrf-2-121 